\section{RIS antenna aided secret key generation for static environment}

Jie Yang, Zheng Wan, Kaizhi Huang, Jianlei Zhao and Xinsheng Ji

Physical layer secret key generation in static environment is challenging due to low secret key capacity. In this paper, a novel secret key generation scheme based on Reconfigurable Intelligent Surface (RIS) antenna is firstly proposed. The traditional antenna is replaced by RIS antenna, and the phase shifts at RIS are tuned randomly in each subframe, which forms a time-varying channel at legitimate users. Then the probed channel coefficients are used to generate the secret key. The analytical expressions of secret key capacity are deduced. We can find that with our scheme, secret key capacity in static environment can be increased to that in fast varying environment, where the frame period is equal to coherent time. Moreover, we can conclude that the scheme has no loss on communication performance, and does not need to change the existing baseband protocol. Simulation results further verify the theoretical analysis.

Introduction: Physical layer secret key generation (SKG) techniques based on wireless channels have been widely studied in recent years, which can provide lightweight encryption for wireless communication systems. However, in static environment, such as the Internet of things (IoT) system, the channel varies slowly, which leads to low secret key rate. Existing work has taken some measures to improve the secret key rate, such as relay aided[1] and user-introduced randomness[2][3] aided SKG schemes. However, to conduct these methods, additional trusted collaboration nodes or the redesigned communication protocols are needed, which is difficult in practical application.

Meanwhile, Reconfigurable Intelligent Surface (RIS), which is a planar array consisting of massive low-cost material elements, has been regarded as a potential key technology in the future network. Each element can individually tune phase shift to allow RIS to carry out different functionalities, such as radiation, reflection of propagating waves. Therefore, as a new promising solution, some literatures have been focused on the RIS aided SKG schemes[4]-[6], where the RIS is utilized as passive reflective surface. In [4], the RIS is deployed near legitimate users, and the reflection shifts are designed with statistical channel information to enhance the signal of legitimate users and weaken that of eavesdroppers, which can increase secret key rate. In [5], a SKG architecture based on a time-dependent randomization of the RIS configuration is proposed. Furthermore, a proof-of-concept system based on a RIS prototype and MIMO radio transceivers is implemented, which verifies key rate can be increased[6]. However, due to the passive characteristic of the reflective surface and large scale propagation loss of electromagnetic waves, when the RIS is not deployed properly, the introduced reflection path has little effect on cascaded channel[7], which results in limited key rate increase.

In fact, active transceiver antenna array is an alternative recent usage of RIS for communication[8]. Compared with conventional antenna arrays, RIS antenna requires much less cost and eliminates the need for complicated corporate feed and/or active phase shifters. Besides, a large number of tunable radiating elements can be packed in a small physical area for a wide range of operating frequencies. In [9], practical mMIMO systems with RIS antenna is proofed to be comparable to that with ideal antenna arrays. By far, RIS antenna has not been discussed in physical layer security.

Motived by above, a RIS antenna aided SKG scheme is firstly proposed. The traditional omnidirectional antenna is replaced with lowcost RIS antenna and a novel transceiver architecture is given. By randomly changing the phase shifts at the RIS antenna, we can construct the agile beampattern to realize the rapid changes of reception channel. Then, we can conclude that the secret key capacity in static environment can be significantly improved.

SYSTEM MODEL: As shown in Fig. 1, we consider a three-node secret key generation model based on RIS antenna. The legitimate users Alice and Bob wish to agree on the secret keys, while Eve is a passive eavesdropper located several wavelengths away from legitimate users and tries to deduce the secret keys. The wireless channel between Alice and

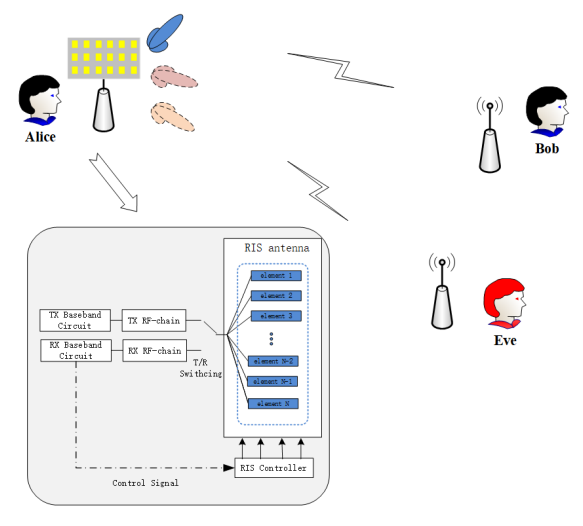

Fig. 1. System model for RIS antenna-aided secret key generation

Bob varies slowly in static enviroment, where the transmission frame period is much less than coherent time.

Unlike the traditional omni-directional antenna, Alice is equipped with a RIS antenna, which is composed of $N$ radiating elements. All elements are cascaded together and connected with a baseband channel. Each element can adjust the phase of the transmitted and received signals, which is equivalent to a directional antenna with variable beampattern. Meanwhile, the RIS controller developed by field-programmable gate array (FPGA) is installed to control the phase shifts of radiating elements in real time. The RIS controller and baseband transceiver establish synchronization by passing control signalling. Bob and Eve are both equipped with a single antenna.

SECRET KEY GENERATION SCHEME: Our proposed SKG scheme consists of three steps: Random phase, Channel probing, and Secret key generation, as shown in Fig.2.

Step 1: Random phase: Before $i$-th round channel probing conducts, the phase shift at each element is randomly generated by Alice through the RIS controller ${ }^{1}$, where the phase shifts at the RIS is denoted as

$$
\mathbf{v}_{i}^{H}=\left[e^{j \theta_{i, 1}}, e^{j \theta_{i, 2}}, \cdots, e^{j \theta_{i, N}}\right]
$$

Step 2: Channel probing: During $i$-th round channel probing, Alice and Bob successively send public pilot signals to each other within one subframe time, and then both parties obtain channel estimates based on the received signals. For ease of analysis, the static channels between users are modelled as Rayleigh channel, the channel from $u$ to $v$ is denoted as $\mathbf{h}_{u v}$, where $u, v \in\{a, b, e\}$ and $\{a, b, e\}$ denote Alice, Bob and Eve, respectively. There is $\mathbf{h}_{u v}=\left[h_{u v, 1}, h_{u v, 2}, \cdots h_{u v, N}\right]^{T}$, where $h_{u v, n} \sim \mathcal{C N}\left(0, \sigma_{h_{u v}}^{2}\right)$.

During the uplink phase, the pilot sequence with $l_{p}$ symbols sent by Bob is denoted as $\sqrt{P}$, where $s$ denotes the pilot sequence and $P$ denotes the transmission power. Then the received signal at Alice can be expressed as

$$
\mathbf{y}_{a}=\sum_{n} h_{b a, n} e^{j \theta_{i, N}} \sqrt{\frac{P}{N}} \mathbf{s}+\mathbf{n}_{a}=\mathbf{v}_{i}^{H} \mathbf{h}_{b a} \sqrt{\frac{P}{N}} \mathbf{s}+\mathbf{n}_{a}
$$

where $\mathbf{n}_{a}$ denotes additive white Gaussian noise (AWGN) at Alice with variance $\sigma_{n}^{2}$.

Similarly, during the downlink phase, with the same configuration, the received signal at Bob or Eve, can be expressed as respectively:

$$
\begin{aligned}
& \mathbf{y}_{b}=\mathbf{v}_{i}^{H} \mathbf{h}_{a b} \sqrt{\frac{P}{N}} \mathbf{s}+\mathbf{n}_{b} \\
& \mathbf{y}_{e}=\mathbf{v}_{i}^{H} \mathbf{h}_{a e} \sqrt{\frac{P}{N}} \mathbf{s}+\mathbf{n}_{e}
\end{aligned}
$$

where $\mathbf{n}_{b}$ and $\mathbf{n}_{e}$ denote AWGN at Bob and Eve with variance $\sigma_{n}^{2}$, respectively.

It can be seen that compared to omni-directional antenna, using RIS antenna is equivalent to phase weighting and combining on the channels. Bob or Alice can then estimate the channel using least square method as

\footnotetext{
${ }^{1}$ It should be noted that the randomness cannot be preset at the transceivers, otherwise some information can be leaked to Eve with some secret means.
} 


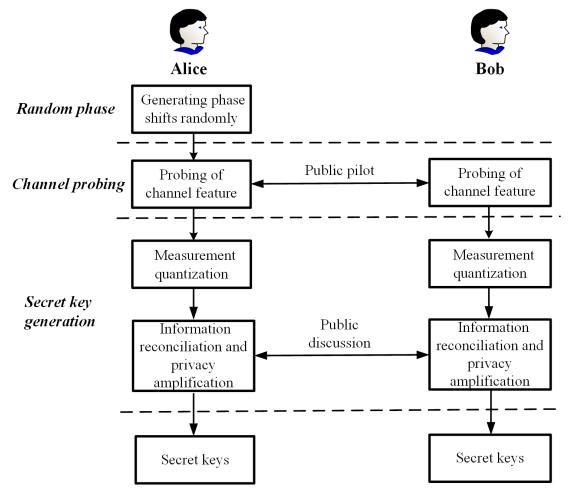

Fig. 2. Key generation procedure

$$
\hat{h}_{u v}=\mathbf{v}_{i}^{H} \mathbf{h}_{u v}+\frac{\sqrt{\frac{P}{N}} \mathbf{s}^{\dagger}}{\| \sqrt{\frac{P}{N}} \mathbf{s}^{2}} \mathbf{n}_{u}
$$

Note that $\mathbf{v}_{i}^{H} \mathbf{h}_{u v}=\sum_{n} h_{u v, n} e^{j \theta_{i, n}}$, and the distribution is hard to derive. Fortunately, since $h_{a b, n} e^{j \theta_{i, n}}, \forall n$ follows the same distribution, when $N$ is large, according to the central limit theorem (CLT), we have

$$
\mathbf{v}_{i}^{H} \mathbf{h}_{u v}=\sum_{n} h_{u v, n} e^{j \theta_{i, n}} \sim \mathcal{C N}\left(0, N \sigma_{u v}^{2}\right)
$$

Therefore, it is easy to know that with RIS antenna, the equivalent channel $\hat{h}_{u v}$ can follow Gaussian distribution, and the estimated variance can be written as

$$
\sigma_{\hat{h}_{u v}}^{2}=\sigma_{\mathbf{v}_{i}^{H} \mathbf{h}_{u v}}^{2}+\frac{\sigma_{n}^{2}}{\left\|\sqrt{\frac{P}{N}} \mathbf{s}\right\|^{2}}=\sigma_{\mathbf{v}_{i}^{H} \mathbf{h}_{u v}}^{2}+\frac{\sigma_{n}^{2}}{\frac{P}{N} l_{p}}
$$

Step 3: Secret key generation: After the channel estimation, uniform quantization is adopted to quantize channel estimates. Then, error correction code is used for information reconciliation to correct mismatch bits. Finally, hash function is utilized for privacy amplification to eliminate leaked information and then the same secret keys between Alice and Bob are generated. More implementation details can refer to [10].

PERFORMANCE ANALYSIS of SKG SCHEME: In this section, the secret key capacity of the proposed scheme is first derived, and then the influence on communication performance and the complexity of the implementation are analyzed.

Secret key capacity analysis: Without losing generality, we only consider extracting secret key from I-channel, and the secret key capacity $C_{S K}$ is

$$
C_{S K}=I\left(\hat{h}_{a b}, \hat{h}_{b a}\right)-I\left(\hat{h}_{a b}, \hat{h}_{a e}\right)
$$

Because $\hat{h}_{a b}$ and $\hat{h}_{b a}$ follow Gaussian distribution, their mutual information can be calculated as

$$
I\left(\hat{h}_{a b}, \hat{h}_{b a}\right)=-\frac{1}{2} \log _{2}\left(1-\rho\left(\hat{h}_{a b}, \hat{h}_{b a}\right)^{2}\right)
$$

where $\rho\left(\hat{h}_{a b}, \hat{h}_{b a}\right)$ denotes the correlation between $\hat{h}_{a b}$ and $\hat{h}_{b a}$ and can be calculated as

$$
\rho\left(\hat{h}_{a b}, \hat{h}_{b a}\right)=\frac{\mathbb{E}\left\{\hat{h}_{a b} \hat{h}_{b a}\right\}}{\sigma_{\hat{h}_{b a}{ } \hat{h}_{a b}}}=\frac{\sigma_{a b}^{2}}{\sigma_{a b}^{2}+\frac{\sigma_{n}^{2}}{P l_{p}}}
$$

Similarly, since the distance between Eve and Alice or Bob is more than half wavelength, $\mathbf{h}_{a b}$ and $\mathbf{h}_{a e}$ are independent and $\rho\left(\hat{h}_{a b}, \hat{h}_{a e}\right)$ can be calculated as

$$
\rho\left(\hat{h}_{a b}, \hat{h}_{a e}\right)=\frac{\mathbb{E}\left\{\hat{h}_{a b} \hat{h}_{a e}\right\}}{\sqrt{\sigma_{\hat{h}_{b a}} \sigma_{\hat{h}_{a e}}}}=0
$$

Then we can have

$$
I\left(\hat{h}_{a b}, \hat{h}_{a e}\right) \stackrel{(a)}{=}-\frac{1}{2} \log _{2}\left(1-\rho\left(\hat{h}_{a b}, \hat{h}_{a e}\right)^{2}\right)=0
$$

From (11), we can see that Eve can not obtain any useful information although the equivalent channel from Alice to Eve $\mathbf{v}_{i}^{H} \mathbf{h}_{a e}$ contains phase shift information. While, it should be noted that equation (a) can hold when the number of radiating units $N$ is large, where $\mathbf{v}_{i}^{H} \mathbf{h}_{a b}$ and $\mathbf{v}_{i}^{H} \mathbf{h}_{a e}$ follow Gaussian distribution. And when $N$ is small, especially considering extreme case $N=1$, equation (a) fails and information will be leaked. It will be further verified by simulation.

Therefore, we can obtain the secret key capacity:

$$
\begin{gathered}
C_{S K}=I\left(\hat{h}_{a b}, \hat{h}_{b a}\right)-I\left(\hat{h}_{a b}, \hat{h}_{a e}\right) \\
=-\frac{1}{2} \log _{2}\left(1-\frac{\sigma_{a b}^{4}}{\left(\sigma_{a b}^{2}+\frac{\sigma_{n}^{2}}{P l_{p}}\right)^{2}}\right)
\end{gathered}
$$

Note that the secret key capacity $C_{S K}^{\prime}$ for fast varying channel, where the frame period is equal to coherent time, is[11]

$$
C_{S K}^{\prime}=-\frac{1}{2} \log _{2}\left(1-\frac{\sigma_{a b}^{4}}{\left(\sigma_{a b}^{2}+\frac{\sigma_{n}^{2}}{P l_{p}}\right)^{2}}\right)
$$

It can be found that $C_{S K}$ and $C_{S K}^{\prime}$ are equal, which means that with RIS antenna, the secrect key capacity under static channel can be increased to the secrect key capacity under fast varying channel. Moreover, $C_{S K}$ is independent of the number of radiating units. And our scheme does not improve the key capacity for fast varying channel.

Impact on communication performance: We consider exploiting the average signal to interference noise ratio (ASINR) as the communication performance metric. The ASINR at Bob can be expressed as:

$$
\mathbb{E}\left(\mathrm{SINR}_{b}\right)=\mathbb{E}\left(\frac{P\left|\sum_{n} h_{a b, n} e^{j \theta_{i, n}}\right|^{2}}{N \sigma_{n}^{2}}\right)
$$

From (5), $\sum_{n} h_{a b, n} e^{j \theta_{i, n}} \sim \mathcal{C N}\left(0, N \sigma_{a b}^{2}\right)$, thus we can have

$$
\mathbb{E}\left(\operatorname{SINR}_{b}\right)=\frac{P \sigma_{a b}^{2}}{\sigma_{n}^{2}}
$$

While, when using omni-directional antenna, the ASINR at Bob can be given by:

$$
\mathbb{E}\left(\operatorname{SINR}_{b}^{\prime}\right)=\mathbb{E}\left(\frac{P\left|h_{a b}\right|^{2}}{\sigma_{n}^{2}}\right)=\frac{P \sigma_{a b}^{2}}{\sigma_{n}^{2}}
$$

Then we can conclude that the communication performance at Bob with RIS antenna is equivalent to that of omnidirectional antenna. Similarly, we can get the same conclusion at Alice. Therefore, the introduction of RIS antenna has no loss on communication performance.

Implementation complexity analysis: Compared with other existing schemes, minor changes for our scheme are needed on existing devices. Firstly, because of the low cost of RIS antenna, the cost of replacing omni-directional antenna with RIS antenna is small. Secondly, in order to ensure that the timing of phase shift modulation is consistent with that of baseband transceiver, the baseband circuit only needs to send the control signal to the RIS controller, without modifying the baseband transceiver module. However, for user-introduced randomness SKG schemes, baseband communication protocols of two sides both need to be redesigned.

Based on above, our proposed scheme can be inserted into the original communication system as an embedded module without modifying protocol and extra performance loss. For the IoT scenarios with central node, our scheme only needs to replace the antenna of the central node and install RIS control module, and the equipment of other nodes does not need to be altered.

SIMULATION RESULTS AND ANALYSIS: In this section, we present results to evaluate the proposed scheme. To ensure the accuracy, we perform Monte Carlo simulation to generate 1000 random channel coefficients in every coherence time (CT) slot. We use MATLAB Information Theoretical Estimator (ITE) Toolbox to numerically compute the mutual information. The parameters are set as follows: $l_{p}=$ $15, \sigma_{n}^{2}=1, \sigma_{a b}^{2}=\sigma_{b a}^{2}=\sigma_{a e}^{2}=5$. The duration of uplink and downlink subframe is set $1 \mathrm{~ms}$. As a comparison, we give the performance without RIS annenta (No IRS).

The performance of two schemes under different $P_{t}$ is shown in Fig.3. It can be seen that with the increase of the CT, the key capacity of the scheme without RIS decreases. While, with RIS antenna, the key capacities under different CT are all improved and are consistent with 


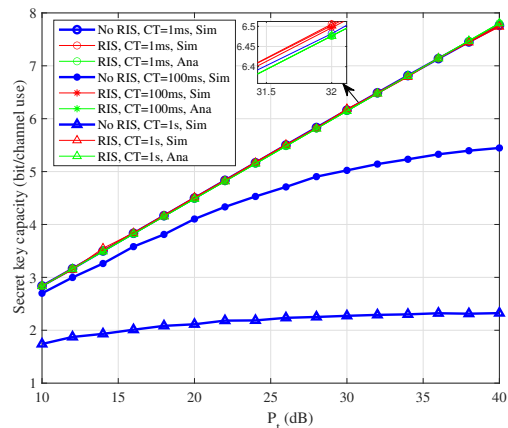

Fig. 3. The secret key capacity verus transmission power $P_{t}(N=30)$

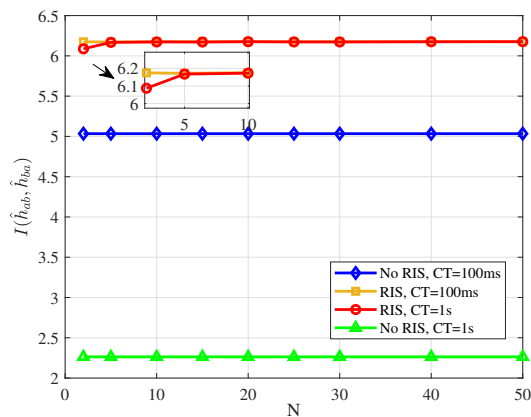

Fig. 4 Mutual information $I\left(\hat{h}_{a b}, \hat{h}_{b a}\right)$ verus the number of radiating units $N\left(P_{t}=30 d B\right)$

the key capacity under fast varying channel, which verifies the theoretical analysis.

From Fig.4, it can be seen that mutual information $I\left(\hat{h}_{a b}, \hat{h}_{b a}\right)$ increases slowly with increasing $N$ and remains almost unchanged when $N \geq 10$. That is to say, although CLT theorem requires a sufficient number of variables theoretically, $N$ is not needed to set too large in fact. Therefore, the RIS antenna with a small number of elements can meet the requirement, which is also consistent with the conclusion where RIS is used as Reflector[5]. This is because although $N$ increases, the transmission power at each channel decreases under the constant total power and the received SINR at each channel decreases.

Fig. 5 gives mutual information $I\left(\hat{h}_{a b}, \hat{h}_{a e}\right)$ verus $N$ under different CT. When the channel is fast varying, the mutual information tends to zero, even if $N$ is small. The reason is that the equivalent channel $\mathbf{v}^{H} \mathbf{h}_{a e}$ always follows Gaussian distribution which is not related to $N$. However, when the channel is static, the mutual information decreases and tends to zero with increasing $N$. This shows that in extreme case, that is, when the channel is constant, although the estimated channels from Alice to Bob and Eve contain the same unidirectional random source, the information leakage can be ignored, which verifies the theoretical analysis.

From Fig.6, it can be seen that the simulation communication performance of two schemes are almost the same and is consistent with

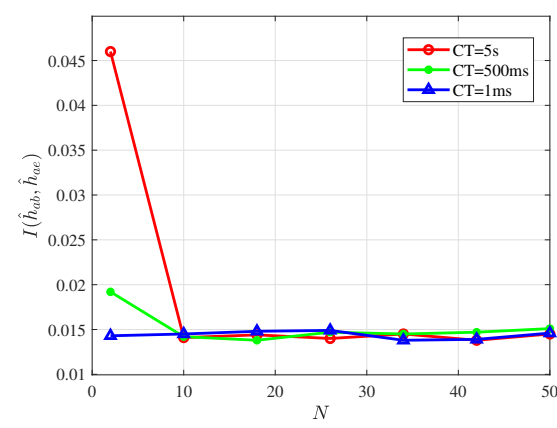

Fig. 5 Mutual information $I\left(\hat{h}_{a b}, \hat{h}_{a e}\right)$ verus the number of radiating units $N\left(P_{t}=30 d B\right)$

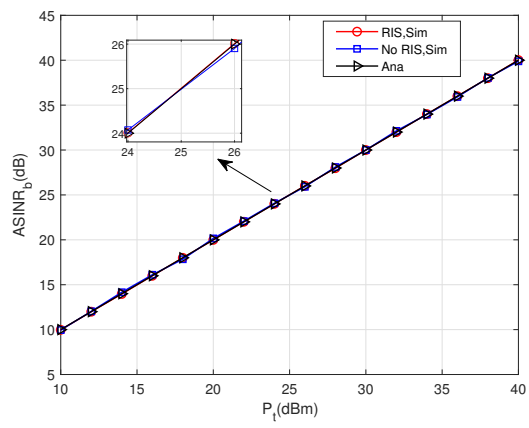

Fig. 6. Communication performance verus transmission power $P_{t}(N=30)$

the theoretical result, which verifies the theoretical analysis. It shows that although the transceiver adopts the random directional antenna, the impact on the communication performance can be ignored.

Conclusion: In this paper, we have presented an investigation of RIS antenna aided physical layer secret key generation. A novel transceiver architecture based on RIS antenna is given. And by adjusting the phase shifts at the radiating elements randomly in each subframe, the secret key capacity can be increased to the same as that in varying environment. Furthermore, compared to existing schemes, our scheme can be conducted with minor changes on original device. This paper only theoretically analyzes the feasibility of the scheme, and the system implementation based on the RIS prototype is left for further study.

Acknowledgment: This work was supported in part by the National Natural Science Foundation of China under Grant 61871404, in part by National Natural Science Foundation Innovative Groups Project of China under Grant 61521003, and in part by key universities and academic disciplines contruction project.

Jie Yang ${ }^{1}$, Zheng Wan ${ }^{1}$, Kaizhi Huang ${ }^{1}$, Jianlei Zhao ${ }^{2}$ and Xinsheng $\mathrm{Ji}^{1}$ (1.Information Engineering University, Zhengzhou 450001, China 2.Network Communication and Security Purple Mountain Laboratory, Nanjing 210000, China)

E-mail: wanzheng18@alumni.hust.edu.cn

\section{References}

1 Xiao, S., Guo, Y., Huang, K., and Jin, L.: High-rate secret key generation aided by multiple relays for Internet of things. Electronics Letters, 53(17), 1198-1200 (2017)

2 L. Jin, S. Zhang, Y. Lou, X. Xu and Z. Zhong.: Secret Key Generation With Cross Multiplication of Two-Way Random Signals. IEEE Access, 7 113065-113080 (2019)

3 N. Aldaghri and H. Mahdavifar.: Physical Layer Secret Key Generation in Static Environments. IEEE Transactions on Information Forensics and Security, 15, 2692-2705(2020)

4 Z. Ji et al.: Secret Key Generation for Intelligent Reflecting Surface Assisted Wireless Communication Networks. IEEE Transactions on Vehicular Technology, 70(1), 1030-1034 (2021)

5 X. Hu, L. Jin, K. Huang, X. Sun, Y. Zhou and J. Qu.: Intelligent Reflecting Surface-Assisted Secret Key Generation with Discrete Phase Shifts in Static Environment. IEEE Wireless Communications Letters( Early Access), 2021

6 Paul Staat, Harald Elders-Boll, Markus Heinrichs and et al.: Intelligent Reflecting Surface-Assisted Wireless Key Generation for Low-Entropy Environments. arXiv 2021.010.06613

7 Q. Wu, S. Zhang, B. Zheng, C. You and R. Zhang.: Intelligent Reflecting Surface-Aided Wireless Communications: A Tutorial. IEEE Transactions on Communications, 69(5), 3313-3351 (2021)

8 N. Shlezinger, G. C. Alexandropoulos, M. F. Imani, Y. C. Eldar and D. R. Smith.: Dynamic Metasurface Antennas for 6G Extreme Massive MIMO Communications. IEEE Wireless Communications, 28(2), 106-113 (2021)

9 H. Wang et al.: Dynamic Metasurface Antennas Based Downlink Massive MIMO Systems. IEEE 20th International Workshop on Signal Processing Advances in Wireless Communications (SPAWC), 1-5(2019)

10 J. Zhang, S. Rajendran, Z. Sun, R. Woods and L. Hanzo.: Physical Layer Security for the Internet of Things: Authentication and Key Generation. IEEE Wireless Communications, 26(5), 92-98 (2019)

11 G. Li, A. Hu, J. Zhang and B. Xiao.: Security Analysis of a Novel Artificial Randomness Approach for Fast Key Generation. IEEE Global Communications Conference, 1-6(2017) 